\title{
The behaviour of free-flowing granular intruders
}

\author{
Edward Wyburn ${ }^{1, \star}$, François Guillard ${ }^{1}$, Benjy Marks ${ }^{1}$, and Itai Einav ${ }^{1,2}$ \\ ${ }^{1}$ Particles and Grains Laboratory, School of Civil Engineering, The University of Sydney, Sydney, New South Wales 2006, Australia \\ ${ }^{2}$ Department of Civil, Environmental \& Geomatic Engineering, Faculty of Engineering Science, University College London, London \\ WC1E 6BT, UK
}

\begin{abstract}
Particle shape affects both the quasi-static and dynamic behaviour of granular media. There has been significant research devoted to the flowability of systems of irregularly shaped particles, as well as the flow of grains around fixed intruders, however the behaviour of free flowing intruders within granular flows remains comparatively unexplored. Here, the effect of the shape of these intruder particles is studied, looking at the kinematic behaviour of the intruders and in particular their tendency of orientation. Experiments are carried out within the Stadium Shear Device, which is a novel apparatus able to continuously apply simple shear conditions to two-dimensional grain analogues. It is found that the intruder shows different behaviour to that of the bulk flow, and that this behaviour is strongly shape dependent. These insights could lead to the development of admixtures that alter the flowability of granular materials.
\end{abstract}

\section{Introduction}

Granular materials exhibit complex behaviour that is not found in other solids or liquids. The properties of the granular mass, whether it be static strength or their subsequent flowing behaviour, are dependent on many factors, including: particle surface roughness, static electricity and cohesive forces, as well as particle size, density, stiffness and shape. Of these factors, shape is particularly important. The majority of granular materials which are produced, collected or conveyed are of an irregular shape. Many tests, both experimental and computational, have used spheres or cylinders due to the simplicity of those shapes [1] with glass beads often chosen for fundamental experiments. There have been studies conducted on systems entirely consisting of irregularly shaped or elongated particles [2-5], as well as the influence of mixtures of regular and elongated particles [6]. The behaviour of a single elongated free flowing intruder within a 2D layer of spheres has also been studied [7]. The elongation of particles in particular introduces many complexities such as the change in jamming transition, altering of shear strength parameters, the orientation of these particles with the principal stress region within sheared assemblies, and the strong dependence on initial conditions [5]. There exist no adequate models to explain these additional factors which are introduced into the rheology of granular materials by anisotropic shaped particles - even for relatively simple shapes such as ellipsoids or rods [5].

There exist many conventional ways of shear testing granular materials, however each of these tests has distinct limitations placed upon it by the geometry. Shear box tests

\footnotetext{
^e-mail: edward.wyburn@ @sydney.edu.au
}

impart simple shear on a sample, however they stipulate the failure plane and have a limited scope for deformation. Triaxial apparatus allow strict control of loading and drainage conditions yet these tests are only able to apply limited deformation and the geometry of the membrane alters as the specimen fails. Couette cells and ring shear are able to continuously shear the sample, however due to their circular geometry contain a radial stress gradient [8]. Preliminary results are provided in this paper which look at the behaviour of rod and teardrop shaped particles of different aspect ratios placed within the 2D Stadium Shear Device (2D SSD - discussed in the next section). The 2D SSD is similar in its loading conditions to the " $1 \gamma 2 \epsilon$ " apparatus [9], which also imparts a shear displacement on 2D grain analogues, yet the 2D SSD is able to perpetually apply simple shear at a constant velocity due to the recirculation of the grains within the curved end zones. The 2D SSD has been used for experiments [10-12] and simulated using spherical and irregular particles [3, 13].

\section{Method}

A schematic of the 2D SSD is shown in Figure 1(a,b). The granular mass has shear imparted upon it by a belt which contains asperities at $14 \mathrm{~mm}$. The rollers directing the belt have constant force springs attached which apply constant confining pressure boundary conditions. A great advantage of the device is the stadium shape which allows for perpetual shear achieved by the gap between the wheels and granular mass allowing for the recirculation of the grains underneath (Figure 1(b)). These tests were all conducted in fixed pressure loading conditions with the same applied pressure and shear rate, which produced an iner- 


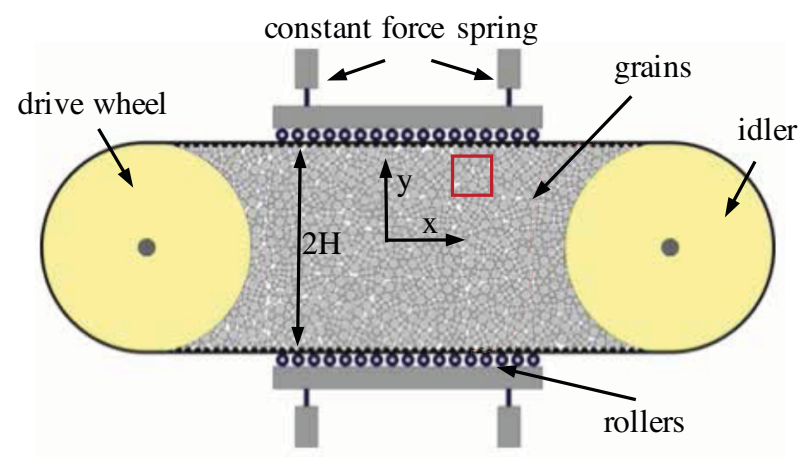

(a)

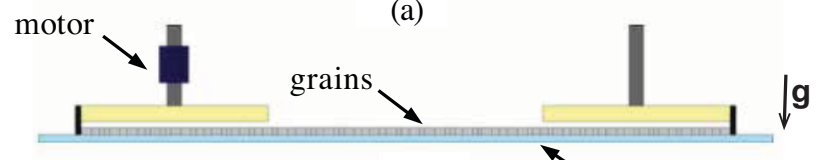

(b)

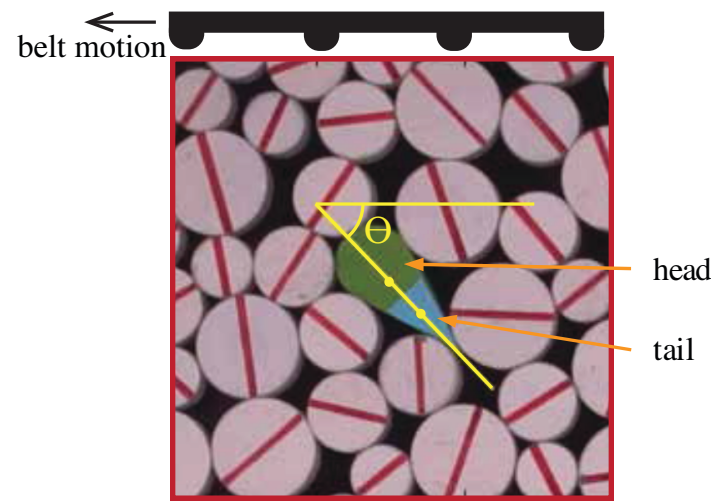

(c)

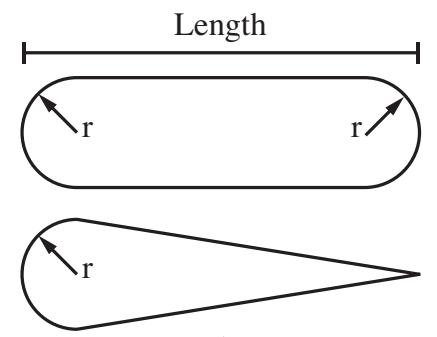

(d)

Figure 1. Diagram of the 2D Stadium Shear Device [12]. (a) top view of the device. (b) elevation view. (c) the long "teardrop" shaped particle sitting within the device surrounded by the typical cylinders. The measured angle is drawn with the yellow line and the two dots correspond to the centroid of the entire particle and the blue area respectively. (d) Diagram showing rod and teardrop intruders.

tial number of approximately 0.04 using an area-weighted average inertial number [14].

The grains within the 2D SSD are $10 \mathrm{~mm}$ high nylon cylinders of three different diameters (12,15 and $20 \mathrm{~mm}$ ) in order to prevent crystallisation from occurring. These grains can be seen in Figure 1(c) along with an intruder particle. These nylon cylinders are durable and have a relatively low friction coefficient of 0.072 against glass [11]. A single 3D printed polylatic acid (PLA plastic) intruder has been placed within the matrix of cylinders for each

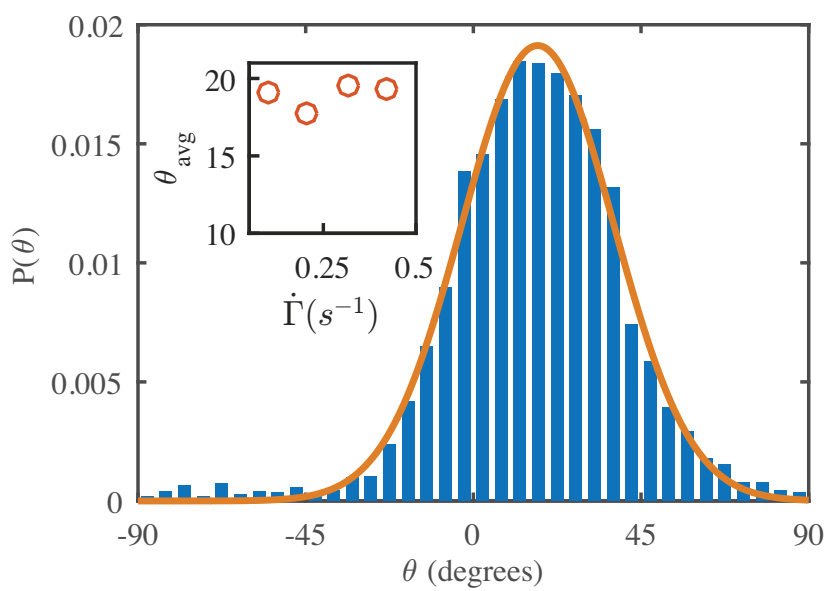

Figure 2. Determination of mean angle $\theta_{\text {avg }}$ for the long rod particle. Here $\dot{\Gamma}$ is the macroscopic shear rate of the device, taken as $V_{\text {belt }} / H$ where $V_{\text {belt }}$ is the belt velocity. Here the angle has been plotted in the interval $\theta \in[-90,90)$ due to the symmetry of the rod shape. A gaussian is fitted to the data and $\theta_{\text {avg }}$ is taken as the value of the peak of the fitted distribution. Inset: $\theta_{\text {avg }}$ as a function of shear rate.

test. Intruders are grouped into two categories, rods and teardrops, and two different length shapes have been analysed for each category.v These intruders are also $10 \mathrm{~mm}$ high and their properties are summarised in Table 1, with aspect ratio defined as the ratio between maximal length and head diameter for all shapes. The first category of intruders can be called rods or capsules. Similar shapes are found widely in nature and industry and have previously received a lot of attention $[6,15,16]$. The second category of intruders, which we have named a teardrop shape, is analogous to a rod particle with one radius equal to zero. These teardrops were produced by taking the convex hull of a cylinder and a point at the desired distance.

Particle tracking velocimetry (PTV) has been used to quantify the full field data. This was performed on videos in $1920 \times 1080$ resolution with a nominal frame rate of 60 fps, which allowed for a displacement of less than $1 / 4$ of the smallest grain diameter between frames. The camera was placed above the device and data was recorded in the central region where pressure is imparted by the springs and the flow is steady. In order to gain significant and smoother statistics for each intruder, since only one was within the mass, the device had to be sheared for at least 200 shear deformations which is many more than was found to be required for the bulk mass. Each intruder particle was tagged with blue tape in order to easily define the tail end and gather the orientation. For the intruders the angle $\theta$ was measured from the centroid of the entire object to the centroid of the tagged tail, which are the yellow dots in Figure 1(c), and gathered in the range of $\theta \in[-180,180)$. For the rod intruders - due to the shape having two axes of symmetry - these angles are doubled by considering an interval of $\theta \in[-90,90)$.

In Figure 2 an example of the method for determination of the mean angle, $\theta_{\text {avg}}$, with a fitted gaussian is 


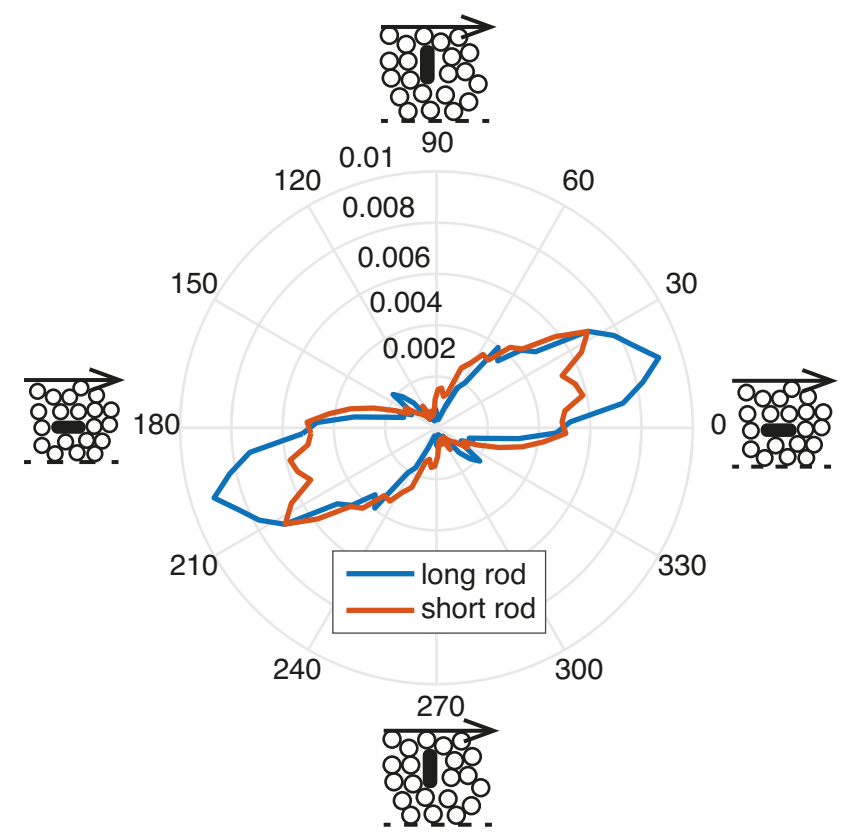

Figure 3. Probability density function $P(\theta)$ of the orientation of the major axis for the rod intruder particles. The radial distance is the probability, and the angle on the graph corresponds to the particle orientation relative to the belt flow. The data has been discretised into bins of 5 degree width to reduce noise. Due to the symmetry of the rod shape this data has been collected in the range of $\theta \in[-90,90)$ and reflected by $180^{\circ}$. The diagrams surrounding the axes show the orientation of the particle with respect to the mean flow direction. A clockwise rotation on the plane corresponds to a clockwise rotation of the particle.

shown. The inset shows tests at four different macroscopic shear rates, with the same confining pressure, which shows the mean orientation's independence from the shear rate which has also been found in previous studies of masses of elongated particles [4-6]. The data presented in the results section was gathered at the highest shear rate shown here. A similar process to find the value of $\theta_{\text {avg }}$ was conducted on the teardrop shaped particles. For those particles instead of taking angles in an interval of $\theta \in[-90,90)$ the range was taken as the 180 degrees of the probability density distribution which yielded the highest probability. For the long teardrop this came to be $\theta \in[142,322)$. The same process was repeated for the short teardrop but the angle probability for this shape does not show a clear orientation preference and results in a poor fit (see Figure 4).

\section{Results}

The graphs in Figures 3 and 4 show the probability density $P(\theta)$ of the orientation of the intruder particles. The measured angles are relative to the belt motion of the half of the flow which the intruder is positioned in. Rod and teardrop shaped particles are both observed to align in a direction that is not parallel to the mean flow direction. The difference in preferred orientation of around 4 degrees between the short and long rod shape is due to a relatively small difference in elongation ( 0.5 rod diameters). When the in-

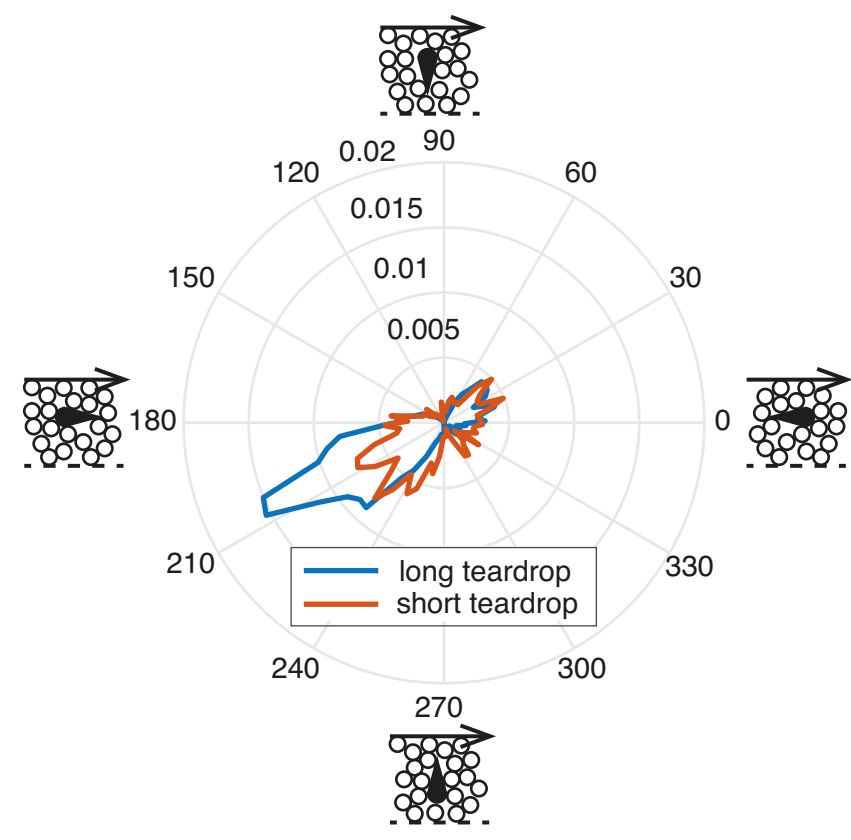

Figure 4. Probability density function $\mathrm{P}(\theta)$ of the orientation of the major axis for the teardrop intruder particles. The radial distance is the probability, and the angle on the graph corresponds to the particle orientation measured from the head to the tail. The data has been discretised into bins of 5 degree width to reduce noise. The diagrams surrounding the axes show the orientation of the particle with respect to the mean flow direction at that angle on the graph. A clockwise rotation on the plane corresponds to a clockwise rotation of the particle.

Table 1. Intruder particle properties

\begin{tabular}{|c|c|c|c|}
\hline Intruder & Head Diameter & Length & $\theta_{\text {avg }}$ \\
\hline Rod - Short & $10 \mathrm{~mm}$ & $30 \mathrm{~mm}$ & $23.1 \cong 203.1^{\circ}$ \\
Rod - Long & $10 \mathrm{~mm}$ & $35 \mathrm{~mm}$ & $19.4 \cong 199.4^{\circ}$ \\
Teardrop - Short & $15 \mathrm{~mm}$ & $22 \mathrm{~mm}$ & $215.5^{\circ}$ \\
Teardrop - Long & $15 \mathrm{~mm}$ & $32 \mathrm{~mm}$ & $207.2^{\circ}$ \\
\hline
\end{tabular}

truder leaves the central zone and enters the areas of circulation below the spindles, mixing occurs due to the strong vortices within this section which often trap the intruder for some time. After leaving these vortices the intruder emerges at a new position and orientation.

The teardrop particles have a strongly asymmetric distribution $\mathrm{P}(\theta)$ due to the shape's single axis of symmetry. These particles interestingly have a tendency to "wedge" where the motion is lead by the tail end of these particles and this pointed end acts as the thin end of a wedge between the other grains. This wedging behaviour is shown by the value of $\theta_{\text {avg }}$ which corresponds to the teardrop shape's head moving within the slower grains and its tail moved by the grains in the swifter moving layer. The wedging mechanism agrees with the findings in [7], where intruder velocity was found to be almost entirely in the direction of the thin end of the intruder particle - however their motion was induced by out-of-plane vertical vibration, which is fundamentally different to shearing the granular mass. There exists a large reduction in wedg- 
ing behaviour for the shorter tailed teardrop. The shorter tailed teardrop is relatively close to a circle, its circularity being 0.68 when defined as the ratio between the radius of the minimum circumscribing circle and the maximum inscribed circle. While there is somewhat of a tendency for wedging to occur with this shape, it does start to approach the behaviour of a circle where there would be expected to be no systematic value for $\theta_{\text {avg }}$. It is uncertain whether a much longer teardrop shape would exhibit this same wedging behaviour, or if due to the larger aspect ratio the larger coordination number would mean that a full rotation of the particle becomes extremely unlikely and the initial condition of intruder orientation is of much more import. It is also uncertain if the mixing beneath the rotors would negate this effect.

The data used in the figures and tables presented has been taken from sections of the flow sufficiently far from the belt and the centre. This was achieved by splitting the flow across the y coordinate into seven portions and ignoring data gathered within the central portion and the portion nearest to each belt. Data near the belt has been ignored due to entrainment occurring within the asperities of the belt which would artificially increase the statistics for a particular angle as the intruder was dragged around at a constant orientation. Since the orientation is taken with respect to the mean flow direction and belt motion, when the intruder crosses the centreline this reverses the angle - which in the case of the teardrop, due to its singlysymmetric cross section and subsequent asymmetric $\mathrm{P}(\theta)$ diagram, makes this data spurious. The reversed information around the centre is still valid for the doubly symmetric rod shapes but has not been included.

The values for $\theta_{\text {avg }}$ of the rods shown here are higher than reported for those of entirely elongated particles, about 10 degrees higher than previous studies where the average angle of rods of aspect ratio 3-3.5 within dynamic sheared assemblies are between approximately 5-10 degrees [5], yet still follow the same trend of increasing $\theta_{\text {avg }}$ with a reduction of aspect ratio. A number of reasons can explain this significantly higher value of $\theta_{\text {avg }}$ for the intruder, however the most likely cause is that the majority of these dynamic simulations or experiments are in $3 \mathrm{D}$ where the extra degree of freedom causes looser packings. In addition it is likely that $\theta_{\text {avg }}$ for the intruder is increased because it is a singular entity within a mass of cylinders which are analogous to rods/teardrops with an aspect ratio of 1 . If the angle of mixtures follows the previously observed trends of inverse proportionality between aspect ratio and $\theta_{\text {avg }}$ [4-6] which was also observed within our tests, it would likely increase the value of $\theta_{\text {avg }}$.

\section{Conclusions}

Preliminary results have been presented which show the behaviour of the intruder particles. Of particular interest was the "wedging" of the teardrop particles, where the thin tail lead the motion by squeezing between other grains. The behaviour of a single rod intruder follows the same trends as previous studies of elongated masses, where $\theta_{\text {avg }}$ is not in the principle flow direction. The presented results are promising and provide good reason to conduct more studies on the effect of elongation as well as the effects of inclusion of multiple intruders on both mean flow fields and the dynamics of the individual intruders.

\section{Acknowledgements}

The authors would like to acknowledge the financial support provided by the Australian Research Council through Discovery Project DP160104310. The authors would also like to thank Garry Towell and Ross Barker for their continued support, as well as Tom Miller for his pioneering work.

\section{References}

[1] GDR MiDi, The European Physical Journal E 14, 341 (2004)

[2] G. Lumay, F. Boschini, K. Traina, S. Bontempi, J.C. Remy, R. Cloots, N. Vandewalle, Powder Technology 224, 19 (2012)

[3] A.X. Jerves, R.Y. Kawamoto, J.E. Andrade, Acta Geotechnica 11, 493 (2016)

[4] T. Börzsönyi, B. Szabó, S. Wegner, K. Harth, J. Török, E. Somfai, T. Bien, R. Stannarius, Phys. Rev. E 86, 051304 (2012)

[5] T. Börzsönyi, R. Stannarius, Soft Matter 9, 7401 (2013)

[6] E. Azéma, I. Preechawuttipong, F. Radjai, Phys. Rev. E 94, 042901 (2016)

[7] N. Kumar, H. Soni, S. Ramaswamy, A.K. Sood, Phys. Rev. E 91, 030102 (2015)

[8] D. Howell, R. Behringer, C. Veje, Physical Review Letters 82, 5241 (1999)

[9] F. Calvetti, G. Combe, J. Lanier, Mechanics of Cohesive-frictional Materials 2, 121 (1997)

[10] T. Miller, P. Rognon, B. Metzger, I. Einav, Phys. Rev. Lett. 111, 058002 (2013)

[11] T. Miller, Ph.D. thesis, The University of Sydney (2014)

[12] P.G. Rognon, T. Miller, I. Einav, Granular Matter 17, 177 (2015)

[13] P.G. Rognon, T. Miller, B. Metzger, I. Einav, Journal of Fluid Mechanics 764 (2015)

[14] P.G. Rognon, J.N. Roux, M. Naaïm, F. Chevoir, Physics of Fluids 19, 058101 (2007)

[15] P.W. Cleary, M.L. Sawley, Applied Mathematical Modelling 26, 89 (2002)

[16] P.W. Cleary, Powder Technology 179, 144 (2008) 